\title{
A Convergence Analysis of Distributed SGD with Communication-Efficient Gradient Sparsification
}

\author{
Shaohuai Shi, Kaiyong Zhao, Qiang Wang, Zhenheng Tang and Xiaowen Chu \\ Department of Computer Science, Hong Kong Baptist University \\ \{csshshi, kyzhao, qiangwang, zhtang, chxw\}@ comp.hkbu.edu.hk
}

\begin{abstract}
Gradient sparsification is a promising technique to significantly reduce the communication overhead in decentralized synchronous stochastic gradient descent (S-SGD) algorithms. Yet, many existing gradient sparsification schemes (e.g., Top- $k$ sparsification) have a communication complexity of $O(k P)$, where $k$ is the number of selected gradients by each worker and $P$ is the number of workers. Recently, the gTop- $k$ sparsification scheme has been proposed to reduce the communication complexity from $O(k P)$ to $O(k \log P)$, which significantly boosts the system scalability. However, it remains unclear whether the gTop- $k$ sparsification scheme can converge in theory. In this paper, we first provide theoretical proofs on the convergence of the gTop- $k$ scheme for non-convex objective functions under certain analytic assumptions. We then derive the convergence rate of gTop- $k \mathrm{~S}$ SGD, which is at the same order as the vanilla minibatch SGD. Finally, we conduct extensive experiments on different machine learning models and data sets to verify the soundness of the assumptions and theoretical results, and discuss the impact of the compression ratio on the convergence performance.
\end{abstract}

\section{Introduction}

Stochastic gradient descent (SGD) algorithms are commonly used for training many machine-learning models. SGD minimizes the objective function $f: \mathbb{R}^{d} \rightarrow \mathbb{R}$ with stochastic gradients $G\left(x_{t}\right)$ using the following update formula:

$$
x_{t+1}=x_{t}-\alpha_{t} G\left(x_{t}\right),
$$

where $x_{t} \in \mathbb{R}^{d}$ is a set of model parameters, and $\alpha_{t} \in \mathbb{R}$ is the step size at iteration $t$. With large-scale models (i.e., $d$ is at the order of millions or even billions) and data sets, distributed synchronous SGD (S-SGD) with data-parallelism is the key technique to reduce the overall training time using multiple computational workers [Goyal et al., 2017; Jia et al., 2018]. Given a cluster with $P$ workers, in the $t^{t h}$ iteration, the $p^{t h}$ worker calculates the gradients $G^{p}\left(x_{t}\right)$ with locally sampled data, and then all workers collaboratively update the model parameters with the aggregated gradients $\frac{1}{P} \sum_{p=1}^{P} G^{p}\left(x_{t}\right)$, i.e.,

$$
x_{t+1}=x_{t}-\alpha_{t} \frac{1}{P} \sum_{p=1}^{P} G^{p}\left(x_{t}\right) .
$$

Ideally, S-SGD with $P$ workers would accelerate the training process by $P$ times. However, the aggregation of gradients requires tremendous data communications among workers, whose time cost becomes significant, especially when the network bandwidth is relatively low [Dean et al., 2012; Shi et al., 2018]. Efficient communication methods have been proposed to alleviate the communication overheads on the system level [Awan et al., 2017; Zhang et al., 2017; Shi et al., 2019a; Chen et al., 2019], while the Top- $k$ sparsification scheme [Chen et al., 2018; Lin et al., 2018] has been proposed to sparsify the gradients to dramatically reduce the communication cost with little impact on the model accuracy on the algorithm level. In Top- $k$ S-SGD, each worker only selects its top- $k$ gradients (in terms of absolute magnitude) to be exchanged with other workers. The update formula becomes:

$$
x_{t+1}=x_{t}-\alpha_{t} \frac{1}{P} \sum_{p=1}^{P} \widetilde{G}^{p}\left(x_{t}\right),
$$

where $\widetilde{G}^{p}\left(x_{t}\right)=\operatorname{TopK}\left(G^{p}\left(x_{t}\right)\right)$ is the sparsified top- $k$ gradients at the $p^{t h}$ worker. Specifically, for a vector $x \in \mathbb{R}^{d}$, $\operatorname{TopK}(x) \in \mathbb{R}^{d}$, and the $i^{\text {th }}(i=1,2, \ldots, d)$ element of $\operatorname{TopK}(x)$ is defined by:

$$
\operatorname{TopK}(x)^{(i)}= \begin{cases}x^{(i)}, & \text { if }\left|x^{(i)}\right|>t h r \\ 0, & \text { otherwise }\end{cases}
$$

where $x^{(i)}$ denotes the $i^{t h}$ element of $x$ and $t h r$ is the $k^{t h}$ largest value of $|x|$. In practice, $k$ can be two to three orders of magnitude smaller than $d$ with little impact on the model accuracy [Aji and Heafield, 2017; Lin et al., 2018; Alistarh et al., 2018], which can dramatically reduce the communication overhead. Some work [Wangni et al., 2018; Stich et al., 2018; Alistarh et al., 2018; Jiang and Agrawal, 2018] has provided the theoretical convergence analysis of Top- $k$ S-SGD under different assumptions.

However, it is noted that the indices of non-zero elements of $\widetilde{G}_{i}^{p}\left(x_{t}\right)$ for different workers are generally inconsistent. 
Therefore, the aggregation of sparsified gradients of Top- $k$ S-SGD from $P$ workers has a communication complexity of $O(k P)$ [Renggli et al., 2018] ${ }^{1}$. Recently, an alternative gradient sparsification scheme named gTop- $k$ [Shi et al., 2019b] has been proposed to reduce the communication complexity from $O(k P)$ to $O(k \log P)$ by using a tree based approximate reduction algorithm. Their empirical studies show that gTop$k$ S-SGD achieves much better training performance than the Top- $k$ scheme on GPU clusters; yet, there is no theoretical justification on the convergence of gTop- $k$ S-SGD.

In this paper, we provide a detailed theoretical analysis on the convergence performance of gTop- $k$ S-SGD on nonconvex problems. We summarize our main contributions as follows:

- Unlike the existing convergence analysis in [Stich et al., 2018; Alistarh et al., 2018] that uses some assumptions on $\sum_{p=1}^{P} \widetilde{G}^{p}\left(x_{t}\right)$ which may not hold in practice, we introduce a relatively weak assumption which can be easily verified through real-world experiments.

- We prove that gTop- $k$ S-SGD provides convergence guarantees for non-convex problems under our analytic assumptions. We conduct extensive experiments on representative deep learning models and data sets to verify the soundness of the assumptions and theoretical results.

- We show that gTop- $k$ S-SGD has the same theoretical convergence rate with vanilla mini-batch SGD with properly chosen learning rates. We also discuss the impact of the compression ratio on the convergence rate through experiments.

\section{Related Work}

There are two main types of communication reduction schemes in S-SGD: gradient quantization and sparsification.

In quantization methods, the exchanged gradients at every iteration can be quantified to a small number of bits (e.g., 2 bits) with error compensation [Alistarh et al., 2017; Wen et al., 2017; Bernstein et al., 2018; Jiang and Agrawal, 2018; Wu et al., 2018; Stich et al., 2018; Wangni et al., 2018; Haddadpour et al., 2019] during communication while keeping the model accuracy nearly unchanged. However, even using only one bit for each gradient, the maximum communication compression ratio is $32 \times$ compared to the 32 -bit counterpart.

In sparsification methods, one can only transmit a small portion of non-zero gradients [Aji and Heafield, 2017; Chen et al., 2018; Lin et al., 2018; Stich et al., 2018; Jiang and Agrawal, 2018; Alistarh et al., 2018; Wangni et al., 2018; Wang et al., 2018] for aggregation so that the communication size can be reduced significantly. Researchers [Aji and Heafield, 2017; Chen et al., 2018; Lin et al., 2018; Renggli et al., 2018] first empirically show the effectiveness of the Top- $k$ or the random- $k$ sparsification in S-SGD with little impact on the model convergence, where $k$ can be only

\footnotetext{
${ }^{1}$ For all-reduce based aggregation, every worker has a communication complexity of $O(k P)$. For parameter-server based aggregation, the parameter server has a communication complexity of $O(k P)$.
}

$0.1 \%$ of the gradient dimension $d$. Some recent work [Stich et al., 2018; Jiang and Agrawal, 2018; Alistarh et al., 2018; Wangni et al., 2018] provides the convergence analysis on Top- $k$ S-SGD under different assumptions. However, even though the Top- $k$ sparsification scheme can zero-out a large number of gradients, it generates the irregular indices among different workers such that the communication complexity is $O(k P)$ for $P$ workers [Renggli et al., 2018], which limits the system scalability. To further reduce the communication complexity, a communication-efficient sparsification scheme named gTop- $k$ has been recently proposed [Shi et al., 2019b]. gTop- $k$ has a communication complexity of $O(k \log P)$ by leveraging a tree structure for gradient communications, and therefore it performs much better than Top- $k$ on large clusters. However, there is no theoretical justification on the convergence of gTop- $k$ S-SGD in [Shi et al., 2019b]. Due to the biased gradients aggregation through the gTop- $k$ sparsification, the theoretical convergence analysis is non-trivial. In this study, we provide the convergence proofs for gTop- $k$ S-SGD, and we conclude that gTop- $k$ S-SGD has the same convergence rate as vanilla S-SGD.

We want to highlight that the existing convergence analysis on Top- $k$ S-SGD [Wangni et al., 2018; Stich et al., 2018; Alistarh et al., 2018; Jiang and Agrawal, 2018] cannot be directly applied to prove the convergence of gTop- $k$ S-SGD. First, gTop- $k$ S-SGD is a biased stochastic compression scheme which is different with the unbiased one in [Wangni et al., 2018]. Second, the analysis in [Stich et al., 2018] is for convex problems, and it requires the sparsification on fully aggregated gradients, while gTop- $k$ S-SGD has no such condition. Third, the analysis in [Jiang and Agrawal, 2018] requires the algorithm to exchange all parameter components in any certain $T$ consecutive iterations, which could also not hold on gTop- $k$ S-SGD since in every iteration only top- $k$ gradients are selected and some very small gradients may not be chosen throughout the training process. Our analysis is closer to the work [Alistarh et al., 2018], but there are three main technical differences. 1) We use a relatively weak analytic assumption on the top- $k$ gradients (and also gTop- $k$ gradients). 2) We eliminate the condition $(k>d / 2)$ that is required in [Alistarh et al., 2018] to guarantee the convergence. 3) We prove the convergence of the gTop- $k$ S-SGD algorithm, and derive the convergence rate, and empirically evaluate the impact of compression ratio on the convergence performance.

\section{The Algorithm of gTop- $k$ S-SGD}

For completeness, in this section we briefly introduce the algorithm of communication-efficient global Top- $k$ (gTop- $k$ ) SSGD proposed in [Shi et al., 2019b]. Before describing the algorithm, we define some notations. Let $v_{t}$ and $\epsilon_{t}^{p}$ denote the local model of each worker and the local gradient residuals of worker $p$ at iteration $t$, respectively. Note that all workers have the consistent model at any iteration. In gTop- $k$ S-SGD, the model is updated by

$$
v_{t+1}=v_{t}-\alpha_{t} \frac{1}{P} \operatorname{gTopK}_{p=1}^{P}\left(G_{t}^{p}\left(v_{t}\right)+\epsilon_{t}^{p}\right),
$$

where $\operatorname{gTopK}_{p=1}^{P}\left(x^{p}\right)=x^{1} \top x^{2} \top \ldots \top x^{P}$, and the operator $\top$ is defined as follows. For any two vectors $x^{i} \in \mathbb{R}^{d}$ and 
$x^{j} \in \mathbb{R}^{d}$,

$$
x^{i} \top x^{j}=\operatorname{mask} \odot\left|\operatorname{TopK}\left(x^{i}\right)+\operatorname{TopK}\left(x^{j}\right)\right|,
$$

where mask $=\left|\operatorname{TopK}\left(x^{i}\right)+\operatorname{TopK}\left(x^{j}\right)\right|>t h r$ and thr is the $k^{\text {th }}$ largest value of $\left|\operatorname{TopK}\left(x^{i}\right)+\operatorname{TopK}\left(x^{j}\right)\right|$. Assume that $x$ is the aggregation result by $\operatorname{gTopK}_{p=1}^{P}\left(x^{p}\right)$, it simultaneously generates a vector of $g M a s k^{p} \in \mathbb{R}^{d}$ which indicates the indices of the selected local values (i.e., $\operatorname{TopK}\left(x^{p}\right)$ ) that contribute to the final $x$. Specifically, the $i^{\text {th }}(i=1,2, \ldots, d)$ element of $g M a s k^{p}$ is defined as

$$
g M a s k^{p,(i)}=\left\{\begin{array}{ll}
1, & \text { If } \operatorname{TopK}\left(x^{p}\right)^{(i)} \text { contributes to } x^{(i)} \\
0, & \text { otherwise }
\end{array} .\right.
$$

The pseudocode of gTop- $k$ S-SGD is shown in Algorithm 1.

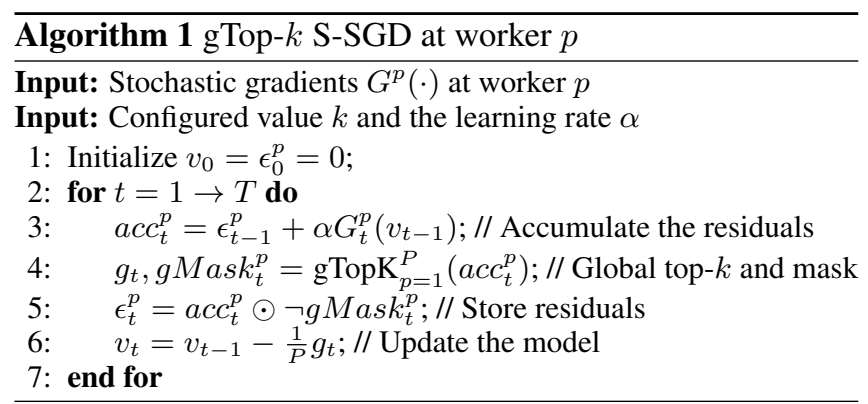

Similar to [Alistarh et al., 2018], we also use $x_{t}$ to denote the auxiliary random variable at iteration $t$, and

$$
x_{t+1}=x_{t}-\alpha G_{t}\left(v_{t}\right) \text {, }
$$

where $G_{t}\left(v_{t}\right)=\frac{1}{P} \sum_{p=1}^{P} G_{t}^{p}\left(v_{t}\right)$ and $x_{0}=0^{d}$. The difference between the auxiliary variable $x_{t}$ and the model variable $v_{t}$ can be represented by

$$
\epsilon_{t}=v_{t}-x_{t} .
$$

According to Algorithm 1, we have $\epsilon_{t}=\frac{1}{P} \sum_{p=1}^{P} \epsilon_{t}^{p}$.

\section{Convergence Analysis}

\subsection{Notations and Assumptions}

We mainly discuss the cases that all the computational workers have a full copy of data. We assume that the gTop- $k$ SSGD is applied to solve the non-convex objective function $f: \mathbb{R}^{d} \rightarrow \mathbb{R}$, which is $L$-Lipschitz smooth, i.e.,

$$
\|\nabla f(x)-\nabla f(y)\| \leq L\|x-y\|, \forall x, y \in \mathbb{R}^{d} .
$$

The sampled stochastic gradients $G(\cdot)$ at every iteration are unbiased, i.e., $\mathbb{E}\left[G\left(v_{t}\right)\right]=\nabla f\left(v_{t}\right)$. We also assume that the second moment of the stochastic gradients is bounded, i.e.,

$$
\mathbb{E}\left[\left\|G_{t}^{p,(i)}(x)\right\|^{2}\right] \leq M^{2}, \forall x \in \mathbb{R}^{d}, \forall t \in \mathbb{N},
$$

where $G_{t}^{p,(i)}(x)$ are the gradients of the $i^{t h}$ sample in a minibatch and $\|\cdot\|$ is $\ell_{2}$-norm. Let $b$ denote the mini-batch size used per worker, and the total mini-batch size with $P$ workers is $B=P b$. The mini-batch setting has $G_{t}^{p}(x)=$ $\frac{1}{b} \sum_{i=1}^{b} G_{t}^{p,(i)}(x)$. Thus, the second moment of the average gradients has a smaller bound, i.e., for any $t \in \mathbb{N}$,

$$
\mathbb{E}\left[\left\|\frac{1}{P} \sum_{p=1}^{P} G_{t}^{p}(x)\right\|^{2}\right] \leq \frac{M^{2}}{P b}=\frac{M^{2}}{B}, \forall x \in \mathbb{R}^{d} .
$$

Assumption 1. The gTopK operator is expected to select $k$ larger values than randomly selecting $k$ values from the accumulated vectors, i.e.,

$$
\begin{aligned}
\mathbb{E}\left[\left\|\frac{1}{P} \sum_{p=1}^{P} x^{p}-\frac{1}{P} \operatorname{gTopK}_{p=1}^{P} x^{p}\right\|^{2}\right] \leq \\
\mathbb{E}\left[\left\|\frac{1}{P} \sum_{p=1}^{P} x^{p}-\operatorname{randomK}\left(\frac{1}{P} \sum_{p=1}^{P} x^{p}\right)\right\|^{2}\right],
\end{aligned}
$$

where $\operatorname{randomK}\left(x^{p}\right) \in \mathbb{R}^{d}$ is a vector whose $k$ elements are randomly selected from $x^{p}$ following a uniform distribution, and the other $d-k$ elements are zeros.

The assumption will be verified by experiments in Section 5. The key ideas of the proofs are 1) We first bound the difference between the model $x_{t}$ without sparsification and the sparsified model $v_{t}$. It enables us to bound the expected sumof-squares of gradients of $f$ so that the convergence is guaranteed [Bottou et al., 2018]. 2) Then we bound the expected average-squared gradients of $f$ with some sufficient conditions to derive the convergence rate.

\subsection{Main Results}

Lemma 1. For any vectors $x^{p} \in \mathbb{R}^{d}, p=1,2, \ldots, P$, and $0<k \leq d$, it holds that

$$
\mathbb{E}\left[\left\|\mid \sum_{p=1}^{P} x^{p}-\operatorname{gTopK}_{p=1}^{P} x^{p}\right\|^{2}\right] \leq\left(1-\frac{k}{d}\right)\left\|\sum_{p=1}^{P} x^{p}\right\|^{2}
$$

Proof. In [Stich et al., 2018], the authors have shown that for any vector $x \in \mathbb{R}^{d}$, it holds

$$
\mathbb{E}\left[\|x-\operatorname{randomK}(x)\|^{2}\right]=\left(1-\frac{k}{d}\right)\|x\|^{2} .
$$

Combined with Assumption 1, we easily obtain

$$
\mathbb{E}\left[\left\|\mid \sum_{p=1}^{P} x^{p}-\operatorname{gTopK}_{p=1}^{P} x^{p}\right\|^{2}\right] \leq\left(1-\frac{k}{d}\right)\left\|\sum_{p=1}^{P} x^{p}\right\|^{2}
$$

Lemma 2. For any iteration $t \geq 1$ :

$$
\mathbb{E}\left[\left\|v_{t}-x_{t}\right\|^{2}\right] \leq \frac{1}{\eta} \sum_{i=1}^{t}(\gamma(1+\eta))^{i} \mathbb{E}\left[\left\|x_{t-i+1}-x_{t-i}\right\|^{2}\right],
$$

where $\gamma=1-\frac{k}{d}, 0<k \leq d$ and $\eta>0$.

Proof. We derive the difference between $v_{t+1}$ and $x_{t+1}$, i.e.,

$$
\mathbb{E}\left[\left\|v_{t+1}-x_{t+1}\right\|^{2}\right]=\mathbb{E}\left[\| \frac{1}{P} \sum_{p=1}^{P}\left(\alpha_{t} G_{t}^{p}\left(v_{t}\right)+\epsilon_{t}^{p}\right)\right.
$$


Proceedings of the Twenty-Eighth International Joint Conference on Artificial Intelligence (IJCAI-19)

$\left.+v_{t}-x_{t}-\epsilon_{t}-\frac{1}{P} \operatorname{gTopK}_{p=1}^{P}\left(\alpha_{t} G_{t}^{p}\left(v_{t}\right)+\epsilon_{t}^{p}\right) \|^{2}\right]=$

$\mathbb{E}\left[\left\|\frac{1}{P} \sum_{p=1}^{P}\left(\alpha_{t} G_{t}^{p}\left(v_{t}\right)+\epsilon_{t}^{p}\right)-\frac{1}{P} \operatorname{gTopK}_{p=1}^{P}\left(\alpha_{t} G_{t}^{p}\left(v_{t}\right)+\epsilon_{t}^{p}\right)\right\|^{2}\right]$

$\leq \gamma\left\|\frac{1}{P} \sum_{p=1}^{P}\left(\alpha_{t} G_{t}^{p}\left(v_{t}\right)+\epsilon_{t}^{p}\right)\right\|^{2}$ (by Lemma 1)

$=\gamma\left\|\alpha_{t} G_{t}\left(v_{t}\right)+v_{t}-x_{t}\right\|^{2}$

$\leq \gamma(1+\eta) \mathbb{E}\left[\left\|v_{t}-x_{t}\right\|^{2}\right]+\gamma\left(1+\frac{1}{\eta}\right) \mathbb{E}\left[\left\|\alpha_{t} G_{t}\left(v_{t}\right)\right\|^{2}\right]$

$=\gamma(1+\eta) \mathbb{E}\left[\left\|v_{t}-x_{t}\right\|^{2}\right]+\gamma\left(1+\frac{1}{\eta}\right) \mathbb{E}\left[\left\|x_{t+1}-x_{t}\right\|^{2}\right]$.

Iterating the above inequality from $i=0 \rightarrow t$ yields:

$$
\begin{aligned}
& \mathbb{E}\left[\left\|v_{t}-x_{t}\right\|^{2}\right] \\
\leq & \gamma\left(1+\frac{1}{\eta}\right) \sum_{i=1}^{t}(\gamma(1+\eta))^{i-1} \mathbb{E}\left[\left\|x_{t-i+1}-x_{t-i}\right\|^{2}\right] \\
= & \frac{1}{\eta} \sum_{i=1}^{t}(\gamma(1+\eta))^{i} \mathbb{E}\left[\left\|x_{t-i+1}-x_{t-i}\right\|^{2}\right] .
\end{aligned}
$$

\section{Corollary 1.}

$$
\mathbb{E}\left[\left\|v_{t}-x_{t}\right\|^{2}\right] \leq \frac{1}{\eta} \sum_{i=1}^{t}(\gamma(1+\eta))^{i} \alpha_{t-i}^{2} \frac{M^{2}}{B} .
$$

Proof. Using Lemma 2 and the bound of (12), we have

$$
\begin{aligned}
\mathbb{E}\left[\left\|v_{t}-x_{t}\right\|^{2}\right] & \leq \frac{1}{\eta} \sum_{i=1}^{t}(\gamma(1+\eta))^{i} \mathbb{E}\left[\left\|x_{t-i+1}-x_{t-i}\right\|^{2}\right] \\
& =\frac{1}{\eta} \sum_{i=1}^{t}(\gamma(1+\eta))^{i} \mathbb{E}\left[\left\|\alpha_{t-i} G_{t-i}\left(v_{t-i}\right)\right\|^{2}\right] \\
& \leq \frac{1}{\eta} \sum_{i=1}^{t}(\gamma(1+\eta))^{i} \alpha_{t-i}^{2} \frac{M^{2}}{B}
\end{aligned}
$$

Theorem 1. Assume that gTop- $k S-S G D$ is applied to minimize the objective function $f$ that satisfies the assumptions in Section 4.1. If one chooses a learning rate schedule such that for any iteration $t>0$ :

$$
\sum_{i=1}^{t}(\gamma(1+\eta))^{i} \frac{\alpha_{t-i}^{2}}{\alpha_{t}} \leq D
$$

for some constant $D>0$, then after running $T$ iterations with Algorithm 1, we have

$$
\begin{aligned}
& \frac{1}{\sum_{t=1}^{T} \alpha_{t}} \sum_{t=1}^{T} \alpha_{t} \mathbb{E}\left[\left\|\nabla f\left(v_{t}\right)\right\|^{2}\right] \leq \\
& \frac{4\left(f\left(x_{0}\right)-f\left(x^{*}\right)\right)}{\sum_{t=1}^{T} \alpha_{t}}+\frac{\left(L+\frac{2 L^{2} D}{\eta}\right) \frac{2 M^{2}}{B} \sum_{t=1}^{T} \alpha_{t}^{2}}{\sum_{t=1}^{T} \alpha_{t}},
\end{aligned}
$$

where $x^{*}$ is the optimal solution to the objective function $f$.
Proof. Under the Assumption of $L$-smooth of $f$, we have

$$
\begin{gathered}
f\left(x_{t+1}\right)-f\left(x_{t}\right) \leq \nabla f\left(x_{t}\right)^{\top}\left(x_{t+1}-x_{t}\right)+\frac{L}{2}\left\|x_{t+1}-x_{t}\right\|^{2} \\
=-\alpha_{t} \nabla f\left(x_{t}\right)^{\top} G_{t}\left(v_{t}\right)+\frac{\alpha_{t}^{2} L}{2}\left\|G_{t}\left(v_{t}\right)\right\|^{2}
\end{gathered}
$$

Taking the expectation at iteration $t$, we have

$$
\begin{aligned}
& \mathbb{E}\left[f\left(x_{t+1}\right)\right]-f\left(x_{t}\right) \\
& \leq-\alpha_{t} \nabla f\left(x_{t}\right)^{\top} \mathbb{E}\left[G_{t}\left(v_{t}\right)\right]+\frac{\alpha_{t}^{2} L}{2} \mathbb{E}\left\|G_{t}\left(v_{t}\right)\right\|^{2} \\
&=-\alpha_{t} \nabla f\left(x_{t}\right)^{\top} \nabla f\left(v_{t}\right)+\frac{\alpha_{t}^{2} L}{2} \mathbb{E}\left[\left\|G_{t}\left(v_{t}\right)\right\|^{2}\right] \\
&=-\frac{\alpha_{t}}{2}\left\|\nabla f\left(x_{t}\right)\right\|^{2}-\frac{\alpha_{t}}{2}\left\|\nabla f\left(v_{t}\right)\right\|^{2} \\
&+\frac{\alpha_{t}}{2}\left\|\nabla f\left(x_{t}\right)-\nabla f\left(v_{t}\right)\right\|^{2}+\frac{\alpha_{t}^{2} L}{2} \mathbb{E}\left[\left\|G_{t}\left(v_{t}\right)\right\|^{2}\right] \\
& \leq-\frac{\alpha_{t}}{2}\left\|\nabla f\left(x_{t}\right)\right\|^{2}+\frac{\alpha_{t} L^{2}}{2}\left\|v_{t}-x_{t}\right\|^{2}+\frac{\alpha_{t}^{2} L}{2} \mathbb{E}\left[\left\|G_{t}\left(v_{t}\right)\right\|^{2}\right] \\
&=-\frac{\alpha_{t}}{2}\left(\left\|\nabla f\left(x_{t}\right)\right\|^{2}+L^{2}\left\|v_{t}-x_{t}\right\|^{2}\right) \\
&+\alpha_{t} L^{2}\left\|v_{t}-x_{t}\right\|^{2}+\frac{\alpha_{t}^{2} L}{2} \mathbb{E}\left[\left\|G_{t}\left(v_{t}\right)\right\|^{2}\right] \\
& \leq-\frac{\alpha_{t}}{2}\left(\left\|\nabla f\left(x_{t}\right)\right\|^{2}+L^{2}\left\|v_{t}-x_{t}\right\|^{2}\right) \\
&+\alpha_{t} L^{2}\left\|v_{t}-x_{t}\right\|^{2}+\frac{\alpha_{t}^{2} L M^{2}}{2 B} .
\end{aligned}
$$

Taking the expectation before $t$, it yields

$$
\begin{aligned}
\mathbb{E}[ & \left.f\left(x_{t+1}\right)\right]-\mathbb{E}\left[f\left(x_{t}\right)\right] \leq \alpha_{t} L^{2} \mathbb{E}\left[\left\|v_{t}-x_{t}\right\|^{2}\right] \\
& +\frac{\alpha_{t}^{2} L M^{2}}{2 B}-\frac{\alpha_{t}}{2} \mathbb{E}\left[\left(\left\|\nabla f\left(x_{t}\right)\right\|^{2}+L^{2}\left\|v_{t}-x_{t}\right\|^{2}\right)\right] \\
\leq & \frac{\alpha_{t} L^{2}}{\eta} \sum_{i=1}^{t}(\gamma(1+\eta))^{i} \alpha_{t-i}^{2} \frac{M^{2}}{B}+\frac{\alpha_{t}^{2} L M^{2}}{2 B} \\
& -\frac{\alpha_{t}}{2} \mathbb{E}\left[\left(\left\|\nabla f\left(x_{t}\right)\right\|^{2}+L^{2}\left\|v_{t}-x_{t}\right\|^{2}\right)\right] \\
= & \frac{\alpha_{t}^{2} L^{2}}{\eta} \sum_{i=1}^{t}(\gamma(1+\eta))^{i} \frac{\alpha_{t-i}^{2}}{\alpha_{t}} \frac{M^{2}}{B}+\frac{\alpha_{t}^{2} L M^{2}}{2 B} \\
& -\frac{\alpha_{t}}{2} \mathbb{E}\left[\left(\left\|\nabla f\left(x_{t}\right)\right\|^{2}+L^{2}\left\|v_{t}-x_{t}\right\|^{2}\right)\right] .
\end{aligned}
$$

Apply (18) to the above inequality, we have

$$
\begin{aligned}
& \mathbb{E}\left[f\left(x_{t+1}\right)\right]-\mathbb{E}\left[f\left(x_{t}\right)\right] \leq\left(L+\frac{2 L^{2} D}{\eta}\right) \frac{M^{2} \alpha_{t}^{2}}{2 B} \\
& -\frac{\alpha_{t}}{2} \mathbb{E}\left[\left(\left\|\nabla f\left(x_{t}\right)\right\|^{2}+L^{2}\left\|v_{t}-x_{t}\right\|^{2}\right)\right] .
\end{aligned}
$$

Then we can obtain

$$
\begin{aligned}
& \alpha_{t} \mathbb{E}\left[\left(\left\|\nabla f\left(x_{t}\right)\right\|^{2}+L^{2}\left\|v_{t}-x_{t}\right\|^{2}\right)\right] \leq \\
& 2\left(\mathbb{E}\left[f\left(x_{t}\right)\right]-\mathbb{E}\left[f\left(x_{t+1}\right)\right]\right)+\left(L+\frac{2 L^{2} D}{\eta}\right) \frac{M^{2} \alpha_{t}^{2}}{B} .
\end{aligned}
$$


Using the $L$-smooth property of $f$, we have

$$
\begin{aligned}
& \left\|\nabla f\left(v_{t}\right)\right\|^{2}=\left\|\nabla f\left(v_{t}\right)-\nabla f\left(x_{t}\right)+\nabla f\left(x_{t}\right)\right\|^{2} \\
& \leq 2\left\|\nabla f\left(v_{t}\right)-\nabla f\left(x_{t}\right)\right\|^{2}+2\left\|\nabla f\left(x_{t}\right)\right\|^{2} \\
& \leq 2 L^{2}\left\|v_{t}-x_{t}\right\|^{2}+2\left\|\nabla f\left(x_{t}\right)\right\|^{2} .
\end{aligned}
$$

Combine with (21), we obtain

$$
\begin{aligned}
& \alpha_{t} \mathbb{E}\left[\left\|\nabla f\left(v_{t}\right)\right\|^{2}\right] \leq 2 \alpha_{t} \mathbb{E}\left[L^{2}\left\|v_{t}-x_{t}\right\|^{2}+\left\|\nabla f\left(x_{t}\right)\right\|^{2}\right] \\
& \leq 4\left(\mathbb{E}\left[f\left(x_{t}\right)\right]-\mathbb{E}\left[f\left(x_{t+1}\right)\right]\right)+2\left(L+\frac{2 L^{2} D}{\eta}\right) \frac{M^{2} \alpha_{t}^{2}}{B} .
\end{aligned}
$$

Summing up the above inequality for $t=1,2, \ldots, T$, we have

$$
\begin{aligned}
& \sum_{t=1}^{T} \alpha_{t} \mathbb{E}\left[\left\|\nabla f\left(v_{t}\right)\right\|^{2}\right] \leq \\
& \quad 4\left(f\left(x_{0}\right)-f\left(x^{*}\right)\right)+2\left(L+\frac{2 L^{2} D}{\eta}\right) \frac{M^{2}}{B} \sum_{t=1}^{T} \alpha_{t}^{2} .
\end{aligned}
$$

By dividing the summation of learning rates, we have:

$$
\begin{aligned}
& \frac{1}{\sum_{t=1}^{T} \alpha_{t}} \sum_{t=1}^{T} \alpha_{t} \mathbb{E}\left[\left\|\nabla f\left(v_{t}\right)\right\|^{2}\right] \leq \\
& \frac{4\left(f\left(x_{0}\right)-f\left(x^{*}\right)\right)}{\sum_{t=1}^{T} \alpha_{t}}+\frac{2\left(L+\frac{2 L^{2} D}{\eta}\right) \frac{M^{2}}{B} \sum_{t=1}^{T} \alpha_{t}^{2}}{\sum_{t=1}^{T} \alpha_{t}}
\end{aligned}
$$

The condition (18) holds if $\gamma(1+\eta)<1$ for both fixed learning rates and diminishing learning rates. To derive the bound of $\eta$, we have

$$
\gamma(1+\eta)=\left(1-\frac{k}{d}\right)(1+\eta)<1 .
$$

Therefore, one should choose $\eta<\frac{k}{d-k}$ to satisfy the above inequality. Theorem 1 implies that Algorithm 1 converges to 0 if $T$ is large enough, when $\alpha_{t}$ is set to satisfy the following conditions:

$$
\lim _{T \rightarrow \infty} \sum_{t=1}^{T} \alpha_{t}=\infty \text { and } \lim _{T \rightarrow \infty} \frac{\sum_{t=1}^{T} \alpha_{t}^{2}}{\sum_{t=1}^{T} \alpha_{t}}=0 .
$$

Corollary 2. Under the assumptions in Theorem 1, if $\tau=$ $\gamma(1+\eta)$ and $\alpha_{t}=\theta \sqrt{B / T}, \forall t>0$, where $\theta>0$ is a constant, we have the convergence rate for Algorithm 1:

$$
\begin{aligned}
& \mathbb{E}\left[\frac{1}{T} \sum_{t=1}^{T}\left\|\nabla f\left(v_{t}\right)\right\|^{2}\right] \\
\leq & \frac{4 \theta^{-1}\left(f\left(x_{0}\right)-f\left(x^{*}\right)\right)+2 \theta L M^{2}}{\sqrt{B T}}+\frac{4 \frac{\tau}{(1-\tau) \eta} L^{2} M^{2} \theta^{2}}{T} .
\end{aligned}
$$

Proof. First we prove that $\alpha_{t}=\theta \sqrt{B / T}$, which is a constant step size (or learning rate), satisfies the condition in (18). We set $\alpha_{t}=\alpha$ for simplification (i.e., $\alpha=\theta \sqrt{B / T}$ ). We have

$\sum_{i=1}^{t}(\gamma(1+\eta))^{i} \frac{\alpha_{t-i}^{2}}{\alpha_{t}}=\sum_{i=1}^{t} \tau^{i} \frac{\alpha_{t-i}^{2}}{\alpha_{t}}=\alpha \sum_{i=1}^{t} \tau^{i}=\alpha \frac{\tau\left(1-\tau^{t}\right)}{1-\tau}$.
Since $0 \leq \tau<1$, we obtain

$$
\lim _{t \rightarrow \infty} \alpha \frac{\tau\left(1-\tau^{t}\right)}{1-\tau}=\frac{\alpha \tau}{1-\tau} .
$$

Therefore, (18) holds by choosing $D=\frac{\alpha \tau}{1-\tau}$. Applying Theorem 1 , we obtain the inequality of the expected averagesquared gradients of $f$, i.e.,

$$
\begin{aligned}
& \mathbb{E}\left[\frac{1}{T} \sum_{t=1}^{T}\left\|\nabla f\left(v_{t}\right)\right\|^{2}\right] \\
& \leq \frac{4\left(f\left(x_{0}\right)-f\left(x^{*}\right)\right)}{\alpha T}+2\left(L+\frac{2 L^{2} D}{\eta}\right) \frac{M^{2} \alpha}{B} \\
& =\frac{4 \theta^{-1}\left(f\left(x_{0}\right)-f\left(x^{*}\right)\right)}{\sqrt{B T}}+\frac{2 L M^{2} \theta}{\sqrt{B T}}+\frac{4 \frac{\tau}{(1-\tau) \eta} L^{2} M^{2} \theta^{2}}{T},
\end{aligned}
$$

which concludes the proof.

From Corollary 2, we can seen that with a properly set learning rate, the gTop- $k$ S-SGD algorithm has a convergence rate of $O\left(\frac{1}{\sqrt{B T}}\right)$, which is the same as that of mini-batch SGD [Dekel et al., 2012]. It also indicates that $k$ has small impact on the convergence rate if $T$ is large enough.

\subsection{Discussion}

In Corollary 2, there are two terms to determine the convergence rate of gTop- $k$ S-SGD. The first term indicates that the convergence rate is affected by the constant $\theta$ and the minibatch size, and the second term indicates that the convergence rate is also affected by both $\theta$ (related to the learning rate) and $\tau$ (related to the compression ratio $\frac{d}{k}$ ). The second term will be dominated by the first term if $T$ is large enough. However, it is not uncommon that a fixed number of iterations is used for training deep neural networks (DNNs) in practice. As a result, although a larger compression ratio leads to less communications overhead, it would enlarge the bound of the convergence rate.

To understand the details, we expand the second term on the right-hand side of (25). Let $c=d / k$ denote the compression ratio, then $\gamma=1-1 / c$ and $\tau=(1-1 / c)(1+\eta)$. Since $\eta$ should satisfy the condition of $\eta<k /(d-k)$, we choose $\eta=k / d=1 / c$. Thus,

$$
\frac{\tau}{(1-\tau) \eta}=\frac{(1-1 / c)(1+\eta)}{\eta-\eta(1-1 / c)(1+\eta)}=c^{3}-c .
$$

Therefore, inequality (25) becomes

$$
\begin{aligned}
& \mathbb{E}\left[\frac{1}{T} \sum_{t=1}^{T}\left\|\nabla f\left(v_{t}\right)\right\|^{2}\right] \\
\leq & \frac{4 \theta^{-1}\left(f\left(x_{0}\right)-f\left(x^{*}\right)\right)+2 \theta L M^{2}}{\sqrt{B T}}+\frac{4 L^{2} M^{2}\left(c^{3}-c\right) \theta^{2}}{T} .
\end{aligned}
$$

The above inequality indicates that given a fixed iteration budget (i.e., $T$ ), a higher compression ratio (c) causes a larger 
bound of the convergence rate. In summary, to achieve a better convergence with a given time budget, one should balance the communication cost and the convergence rate. We will further evaluate the impact of the compression ratio on the convergence performance through experiments in Section 5.

\section{Experiments}

\subsection{Experimental Settings}

Our experimental settings cover three deep learning applications. 1) Image classification: Two popular DNNs, VGG-16 [Simonyan and Zisserman, 2014] and ResNet-20 [He et al., 2016], are used for evaluation on the data set of Cifar- $10^{2}$ which consists of 50000 training images. 2) Language model: A 2-layer LSTM model (LSTM-PTB) with 1500 hidden units per layer is adopted for evaluation on the data set of PTB [Marcus et al., 1993], which contains 923000 training words. 3) Speech recognition: A 5-layer LSTM model (LSTM-AN4) with 800 hidden units per layer is used for evaluation on AN4 [Acero, 1990], which contains 948 training utterances. In all training models, we exploit the warmup strategy in gTop- $k$ S-SGD on the 4-worker distributed environment. The baselines are evaluated using S-SGD without gradient sparsification. The main hyper-parameters adopted in evaluation are shown in Table 1.

\begin{tabular}{|l|l|l|l|}
\hline DNN & $B$ & Initial $\alpha$ & \# of epochs \\
\hline \hline VGG-16 & 512 & 0.1 & 140 \\
\hline ResNet-20 & 128 & 0.1 & 140 \\
\hline LSTM-PTB & 400 & 30 & 40 \\
\hline LSTM-AN4 & 32 & 0.0002 & 80 \\
\hline
\end{tabular}

Table 1: Hyper-parameters for different DNNs

\subsection{Verification of Assumption and Convergences}

We verify Assumption 1 empirically by training DNNs with gTop- $k$ S-SGD. During the training process, we measure

$$
\delta=\frac{\mathbb{E}\left[\left\|\frac{1}{P} \sum_{p=1}^{P} x^{p}-\frac{1}{P} \operatorname{gTopK}_{p=1}^{P} x^{p}\right\|^{2}\right]}{\mathbb{E}\left[\left\|\frac{1}{P} \sum_{p=1}^{P} x^{p}-\operatorname{randomK}\left(\frac{1}{P} \sum_{p=1}^{P} x^{p}\right)\right\|^{2}\right]},
$$

where $x^{p}=G_{t}^{p}\left(v_{t}\right)+\epsilon_{t}^{p}$ and $k=0.001 \times d$ (i.e., $\left.c=1000\right)$. Assumption 1 holds if $\delta \leq 1$. The measurements of $\delta$ corresponded with the training losses on the evaluated DNNs are shown in Fig. 1. It can be seen that we always have $\delta<1$, which verifies the soundness of Assumption 1. In Fig. 1, the convergences of gTop- $k$ S-SGD are nearly consistent with SSGD, which validates our theoretical results and shows that gTop- $k$ S-SGD can converge as fast as S-SGD.

\subsection{Convergence Rate v.s. Compression Ratio}

The second term in inequality (27) indicates that the convergence rate may be degraded by the compression ratio $c$. We evaluate the sensitivity of the convergence rates to $c$ on training DNNs without changing hyper-parameters including the total number of iterations (i.e., a fixed number of epochs).

\footnotetext{
${ }^{2}$ https://www.cs.toronto.edu/ kriz/cifar.html
}

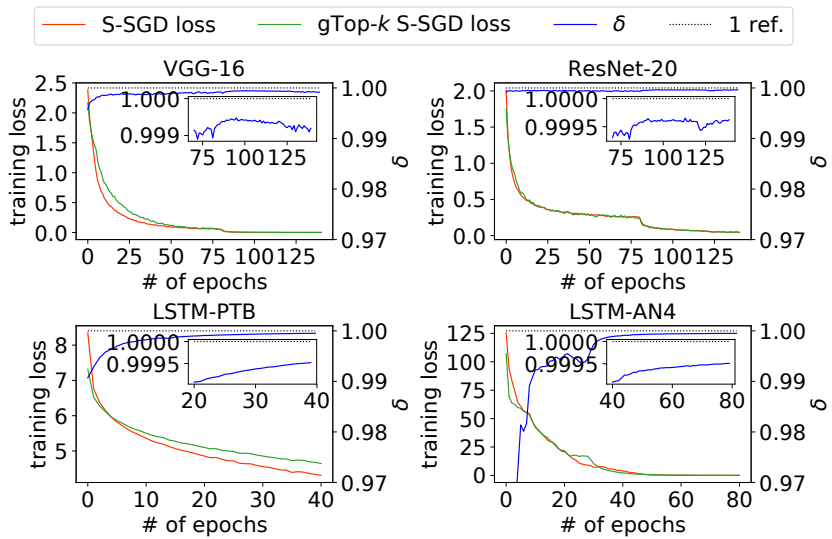

Figure 1: The measurements of $\delta$ and convergences on different DNNs with a compression ratio $c=1000$

The results are shown in Fig. 2, which shows that with larger $c$, the convergence of the models would slowdown. Therefore, with large compression ratios, there is a trade-off between the communication size, which is directly related to the iteration time, and the convergence rate.
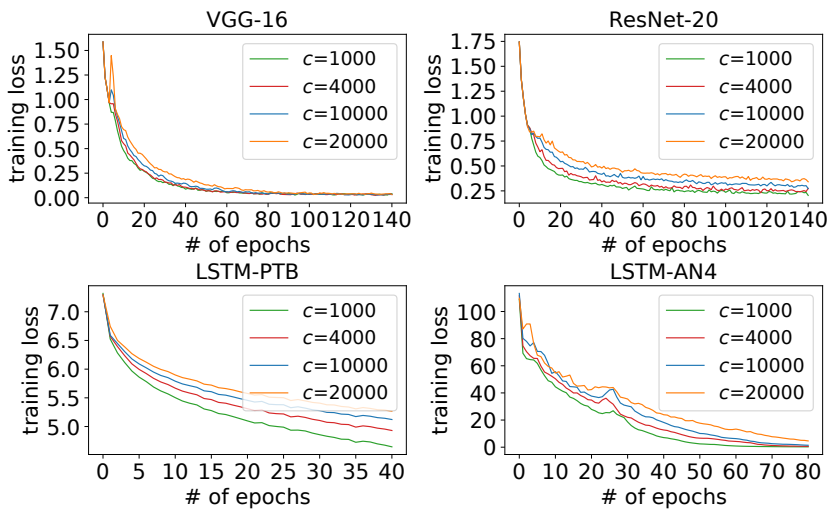

Figure 2: The convergences with different compression ratios

\section{Conclusion}

Top- $k$ gradient sparsification is crucial for reducing the communication size in distributed S-SGD. The gTop- $k$ scheme is a more communication efficient scheme than Top- $k$ for gradient sparsification. In this study, we present a detailed convergence analysis for gTop- $k$ S-SGD under some analytical assumptions, and we derive its convergence rate. Our theoretical results conclude that gTop- $k$ S-SGD provides convergence guarantees for non-convex objective functions and it has the same convergence rate with vanilla mini-batch SGD with properly chosen learning rates. We derive and evaluate the impact of compression ratios on the convergence performance. We finally conduct experiments to verify the soundness of the analytical assumption and theoretical results.

\section{Acknowledgements}

The research was supported by Hong Kong RGC GRF grant HKBU 12200418. 


\section{References}

[Acero, 1990] Alejandro Acero. Acoustical and environmental robustness in automatic speech recognition. In ICASSP, 1990.

[Aji and Heafield, 2017] Alham Fikri Aji and Kenneth Heafield. Sparse communication for distributed gradient descent. In EMNLP, pages 440-445, 2017.

[Alistarh et al., 2017] Dan Alistarh, Demjan Grubic, Jerry $\mathrm{Li}$, et al. QSGD: Communication-efficient SGD via gradient quantization and encoding. In NIPS, pages 1709-1720, 2017.

[Alistarh et al., 2018] Dan Alistarh, Torsten Hoefler, Mikael Johansson, et al. The convergence of sparsified gradient methods. In NeurIPS, pages 5977-5987, 2018.

[Awan et al., 2017] Ammar Ahmad Awan, Khaled Hamidouche, Jahanzeb Maqbool Hashmi, et al. S-Caffe: Codesigning MPI runtimes and Caffe for scalable deep learning on modern GPU clusters. In PPoPP, pages 193-205. ACM, 2017.

[Bernstein et al., 2018] Jeremy Bernstein, Yu-Xiang Wang, Kamyar Azizzadenesheli, et al. signSGD: compressed optimisation for non-convex problems. In ICML, pages 559568, 2018

[Bottou et al., 2018] Léon Bottou, Frank E Curtis, and Jorge Nocedal. Optimization methods for large-scale machine learning. Siam Review, 60(2):223-311, 2018.

[Chen et al., 2018] Chia-Yu Chen, Jungwook Choi, Daniel Brand, et al. Adacomp: Adaptive residual gradient compression for data-parallel distributed training. In $A A A I$, pages 2827-2835, 2018.

[Chen et al., 2019] Chen Chen, Wei Wang, and Bo Li. Round-robin synchronization: Mitigating communication bottlenecks in parameter servers. In IEEE INFOCOM, 2019.

[Dean et al., 2012] Jeffrey Dean, Greg Corrado, Rajat Monga, et al. Large scale distributed deep networks. In NIPS, pages 1223-1231, 2012.

[Dekel et al., 2012] Ofer Dekel, Ran Gilad-Bachrach, Ohad Shamir, et al. Optimal distributed online prediction using mini-batches. Journal of Machine Learning Research, 13(Jan):165-202, 2012.

[Goyal et al., 2017] Priya Goyal, Piotr Dollár, Ross Girshick, et al. Accurate, large minibatch SGD: training ImageNet in 1 hour. arXiv preprint arXiv:1706.02677, 2017.

[Haddadpour et al., 2019] Farzin Haddadpour, Mohammad Mahdi Kamani, Mehrdad Mahdavi, et al. Trading redundancy for communication: Speeding up distributed SGD for non-convex optimization. In ICML, pages 2545-2554, 2019.

[He et al., 2016] Kaiming He, Xiangyu Zhang, Shaoqing Ren, et al. Deep residual learning for image recognition. In $C V P R$, pages 770-778, 2016.

[Jia et al., 2018] Xianyan Jia, Shutao Song, Shaohuai Shi, et al. Highly scalable deep learning training system with mixed-precision: Training ImageNet in four minutes. In NeurIPS Workshop MLSys, 2018.

[Jiang and Agrawal, 2018] Peng Jiang and Gagan Agrawal. A linear speedup analysis of distributed deep learning with sparse and quantized communication. In NeurIPS, pages 2530-2541, 2018.

[Lin et al., 2018] Yujun Lin, Song Han, Huizi Mao, et al. Deep gradient compression: Reducing the communication bandwidth for distributed training. In ICLR, 2018.

[Marcus et al., 1993] Mitchell P Marcus, Mary Ann Marcinkiewicz, and Beatrice Santorini. Building a large annotated corpus of English: The Penn Treebank. Computational linguistics, 19(2):313-330, 1993.

[Renggli et al., 2018] Cèdric Renggli, Dan Alistarh, and Torsten Hoefler. SparCML: High-performance sparse communication for machine learning. arXiv preprint arXiv:1802.08021, 2018.

[Shi et al., 2018] Shaohuai Shi, Wang Qiang, and Xiaowen Chu. Performance modeling and evaluation of distributed deep learning frameworks on GPUs. In IEEE DataCom, pages 949-957, 2018.

[Shi et al., 2019a] Shaohuai Shi, Xiaowen Chu, and Bo Li. MG-WFBP: Efficient data communication for distributed synchronous SGD algorithms. In IEEE INFOCOM, 2019.

[Shi et al., 2019b] Shaohuai Shi, Qiang Wang, Kaiyong Zhao, et al. A distributed synchronous SGD algorithm with global Top- $k$ sparsification for low bandwidth networks. In IEEE ICDCS, 2019.

[Simonyan and Zisserman, 2014] Karen Simonyan and Andrew Zisserman. Very deep convolutional networks for large-scale image recognition. arXiv preprint arXiv:1409.1556, 2014.

[Stich et al., 2018] Sebastian U Stich, Jean-Baptiste Cordonnier, and Martin Jaggi. Sparsified SGD with memory. In NeurIPS, pages 4452-4463, 2018.

[Wang et al., 2018] Hongyi Wang, Scott Sievert, Shengchao Liu, et al. Atomo: Communication-efficient learning via atomic sparsification. In NeurIPS, pages 9850-9861, 2018.

[Wangni et al., 2018] Jianqiao Wangni, Jialei Wang, Ji Liu, et al. Gradient sparsification for communication-efficient distributed optimization. In NeurIPS, pages 1306-1316, 2018.

[Wen et al., 2017] Wei Wen, Cong Xu, Feng Yan, et al. Terngrad: Ternary gradients to reduce communication in distributed deep learning. In NIPS, pages 1509-1519, 2017.

[Wu et al., 2018] Jiaxiang Wu, Weidong Huang, Junzhou Huang, et al. Error compensated quantized SGD and its applications to large-scale distributed optimization. In ICML, pages 5321-5329, 2018.

[Zhang et al., 2017] Hao Zhang, Zeyu Zheng, Shizhen Xu, et al. Poseidon: an efficient communication architecture for distributed deep learning on GPU clusters. In USENIX ATC, pages 181-193, 2017. 